Kragujevac Journal of Mathematics

Volume 46(1) (2022), Pages 7-19.

\title{
NEW INTEGRAL EQUATIONS FOR THE MONIC HERMITE POLYNOMIALS
}

\author{
KARIMA ALI KHELIL ${ }^{1}$, RIDHA SFAXI ${ }^{2}$, AND AMMAR BOUKHEMIS ${ }^{1}$
}

\begin{abstract}
In this article, we are study the question of existence of integral equation for the monic $\mathcal{H}$ ermite polynomials $H_{n}$, where the intervening real function does not depend on the index $n$, well-known by the linear functional $\mathscr{W}_{x}$ given by its moments $H_{n}(x)=\left\langle\mathscr{W}_{x}, t^{n}\right\rangle, n \geq 0,|x|<\infty$. Also, we obtain some properties of the zeros of this intervening function. Furthermore, we obtain an integral representation of the Dirac mass $\delta_{x}$, for every real number $x$.
\end{abstract}

\section{INTRODUCTION}

Given two sequences $\left\{B_{n}\right\}_{n \geq 0}$ and $\left\{Q_{n}\right\}_{n \geq 0}$ of normalized polynomials with real coefficients, with one real variable $x$ and where $\operatorname{deg} B_{n}=\operatorname{deg} Q_{n}=n$, for every integer $n \geq 0$. The problem of integral equation between these two polynomial sequences consists in finding a real function $u(\cdot, t)$ defined in $I \times \mathbb{R}$, where $I \subset \mathbb{R}=]-\infty,+\infty$, and satisfying the condition:

$$
\int_{-\infty}^{\infty} u(x, t) t^{n} \mathrm{~d} t<\infty, \quad n \geq 0, x \in I,
$$

such that

$$
B_{n}(x)=\int_{-\infty}^{\infty} u(x, t) Q_{n}(t) \mathrm{d} t, \quad n \geq 0, x \in I .
$$

When $Q_{n}(x)=x^{n}$, for all integer $n \geq 0$, i.e.,

$$
B_{n}(x)=\int_{-\infty}^{\infty} u(x, t) t^{n} \mathrm{~d} t, \quad n \geq 0, x \in I,
$$

Key words and phrases. Linear functional, integral equation, integral representation on the real line, Hermite polynomials, Dawson function, Dirac mass.

2010 Mathematics Subject Classification. Primary: 33C45, 42C05.

DOI 10.46793/KgJMat2201.007K

Received: January 19, 2019.

Accepted: July 25, 2019. 
we recognize the usual integral representation of the polynomial sequence $\left\{B_{n}\right\}_{n \geq 0}$, called here by the canonical-integral representation of $\left\{B_{n}\right\}_{n \geq 0}$. When $Q_{n}(x)=B_{n}(x)$, for all integer $n \geq 0$, i.e.,

$$
B_{n}(x)=\int_{-\infty}^{\infty} u(x, t) B_{n}(t) \mathrm{d} t, \quad n \geq 0, x \in I,
$$

it is appropriate to say that it is an auto-integral representation of $\left\{B_{n}\right\}_{n \geq 0}$.

In fact, this kind of integral equation is of great relevance in the theory of orthogonal polynomials as well as the moment theory and their applications, [8,9,3,15]. For this reason-in the past as nowadays has attracted the attention of many authors; see, for instance, $[5,6,7,12,4,1,10,11]$. Based on the principle that the terms of any sequence of complex numbers are the moments of a unique linear functional on polynomials, the study of such linear functionals accurate some hypergeometric properties of such sequences, $[2,13,14]$.

In this work, we are interested by the normalized Hermite polynomial sequence $\left\{H_{n}\right\}_{n \geq 0}$. Recall that $\left\{H_{n}\right\}_{n \geq 0}$ is orthogonal with respect to a linear functional on polynomials, namely $\mathscr{H}$ and well-known by its integral representation on the real line $[10]$

$$
\langle\mathscr{H}, p\rangle=\frac{1}{\sqrt{\pi}} \int_{-\infty}^{\infty} p(t) e^{-t^{2}} \mathrm{~d} t, \quad p \in \mathbb{P},
$$

where $\mathbb{P}$ is the vector space of polynomials in one variable with real coefficients and $\mathbb{P}^{\prime}$ its algebraic dual space. Notice that $\langle u, p\rangle$ is the action of a linear functional $u \in \mathbb{P}^{\prime}$ on $p \in \mathbb{P}$ and by $(u)_{n}:=\left\langle u, t^{n}\right\rangle, n \geq 0$, the moments of $u$ with respect to the canonical sequence $\left\{t^{n}\right\}_{n>0}$. For any $u$ in $\mathbb{P}^{\prime}$, any $q$ in $\mathbb{P}$ and any complex numbers $a, b, c$ with $a \neq 0$, recall that $D u=u^{\prime}, q u, h_{a} u$ and $\tau_{b} u$, be respectively, the derivative, the left multiplication, the homothetic and the translation of the linear functionals defined by duality [9]:

$$
\begin{aligned}
\left\langle u^{\prime}, f\right\rangle & :=-\left\langle u, f^{\prime}\right\rangle, \\
\langle q u, f\rangle & :=\langle u, q f\rangle \\
\left\langle h_{a} u, f\right\rangle & :=\left\langle u, h_{a} f\right\rangle=\langle u, f(a x)\rangle, \\
\left\langle\tau_{-b} u, f\right\rangle & :=\left\langle u, \tau_{b} f\right\rangle=\langle u, f(x-b)\rangle, \quad f \in \mathbb{P} .
\end{aligned}
$$

The linear functional $\mathscr{H}$ is normalized, i.e., $(\mathscr{H})_{0}=1$. It satisfies the following Pearson equation [10]:

$$
\mathscr{H}^{\prime}+2 x \mathscr{H}=0_{\mathbb{P}^{\prime}} .
$$

The moments of $\mathscr{H}$ are given by

$$
(\mathscr{H})_{n}=\frac{n !}{2^{n+1} \Gamma\left(\frac{n}{2}+1\right)}\left(1+(-1)^{n}\right), \quad n \geq 0 .
$$

This leads to the following integral representation of the moments of $\mathscr{H}$

$$
\frac{n !}{2^{n+1} \Gamma\left(\frac{n}{2}+1\right)}\left(1+(-1)^{n}\right)=\frac{1}{\sqrt{\pi}} \int_{-\infty}^{\infty} t^{n} e^{-t^{2}} \mathrm{~d} t, \quad n \geq 0 .
$$


The normalized Hermite polynomial $H_{n}$ can be represented in terms of a definite integral containing the real variable $x$ as parameter [8]

$$
H_{n}(x)=\frac{e^{x^{2}}}{\sqrt{\pi}} \int_{-\infty}^{\infty}(-i t)^{n} e^{-t^{2}+2 i t x} \mathrm{~d} t, \quad n \geq 0,|x|<\infty .
$$

Equivalently,

$$
H_{n}(x)=\int_{0}^{\infty} h_{n}(t, x) t^{n} \mathrm{~d} t, \quad n \geq 0,|x|<\infty
$$

where the intervening real function $h_{n}(t, \cdot)$ depends on the integer $n$, and given by

$$
h_{n}(t, x)=\frac{2}{\sqrt{\pi}} e^{x^{2}-t^{2}} \cos \left(2 t x+n \frac{\pi}{2}\right) .
$$

The polynomial $H_{n}$ satisfies the following integral equation [8]

$$
H_{n}(x)=\frac{(-i)^{n}}{\sqrt{2 \pi}} e^{\frac{x^{2}}{2}} \int_{-\infty}^{\infty} e^{-\frac{t^{2}}{2}+i t x} H_{n}(t) \mathrm{d} t, \quad n \geq 0,|x|<\infty .
$$

Equivalently,

$$
H_{n}(x)=\int_{0}^{\infty} r_{n}(t, x) H_{n}(t) \mathrm{d} t, \quad n \geq 0,|x|<\infty,
$$

where the real function $r_{n}(t, \cdot)$ depends on the integer $n$, and given by

$$
r_{n}(t, x)=\frac{1}{\sqrt{2}} h_{n}\left(\frac{t}{\sqrt{2}}, \frac{x}{\sqrt{2}}\right) .
$$

The main purpose of this work is to give two new integral equations for the polynomial sequence $\left\{H_{n}\right\}_{n \geq 0}$, where the intervening real functions do not depend on the integer $n$. In summary, we are going to establish the following.

- The canonical-integral representation:

$$
H_{n}(x)=\int_{-\infty}^{\infty} U(t-x) t^{n} \mathrm{~d} t, \quad n \geq 0,|x|<\infty,
$$

where

$$
\begin{aligned}
U(t) & =S^{-1} e^{t^{2}} \int_{|t|}^{\infty} e^{-y^{2}} e^{-y^{\frac{1}{4}}} \sin y^{\frac{1}{4}} \mathrm{~d} y \\
S & =\int_{-\infty}^{\infty} e^{\xi^{2}} \int_{|\xi|}^{\infty} e^{-y^{2}} e^{-y^{\frac{1}{4}}} \sin y^{\frac{1}{4}} \mathrm{~d} y \mathrm{~d} \xi>0 .
\end{aligned}
$$

- The auto-integral representation:

$$
H_{n}(x)=\int_{-\infty}^{\infty} V(t-x) H_{n}(t) \mathrm{d} t, \quad n \geq 0,|x|<\infty,
$$

where

$$
V(t)= \begin{cases}\frac{e^{-t^{\frac{1}{4}}} \sin \left(t^{\frac{1}{4}}\right)}{\pi t}, & \text { if } t>0, \\ 0, & \text { if } t \leq 0 .\end{cases}
$$




\section{New Canonical-Integral Representation of $\left\{H_{n}\right\}_{n \geq 0}$}

First, let us recall some properties of $\left\{H_{n}\right\}_{n \geq 0},[8,10]$.

- The Taylor expansion:

$$
H_{n}(x)=\sum_{k=0}^{\left[\frac{n}{2}\right]} \frac{(-1)^{k} n !}{2^{2 k} k !(n-2 k) !} x^{n-2 k}, \quad n \geq 0 .
$$

- The symmetry property:

$$
H_{n}(-x)=(-1)^{n} H_{n}(x), \quad n \geq 0 .
$$

- The Appel property:

$$
H_{n}^{\prime}(x)=n H_{n-1}(x), \quad n \geq 0, H_{-1}(x)=0 .
$$

- The three-terms-recurrence relation:

$$
\left\{\begin{array}{l}
H_{-1}(x)=0, \quad H_{0}(x)=1, \\
H_{n+1}(x)=x H_{n}(x)-\frac{n}{2} H_{n-1}(x), \quad n \geq 0 .
\end{array}\right.
$$

Next, let $\mathscr{W}_{x}$ be the linear functional on polynomials and given by its moments

$$
H_{n}(x)=\left\langle\mathscr{W}_{x}, t^{n}\right\rangle, \quad n \geq 0,|x|<\infty .
$$

From (2.1) and (2.3), we show that

$$
\mathscr{W}_{-x}=h_{-1}\left(\mathscr{W}_{x}\right), \quad|x|<\infty .
$$

From (2.2) and (2.3), the linear functional $\mathscr{W}_{x}$ satisfies

$$
\left(\mathscr{W}_{x}\right)_{0}=1, \quad \mathscr{W}_{x}^{\prime}-2(t-x) \mathscr{W}_{x}=0, \quad|x|<\infty .
$$

Lemma 2.1. For any real number $x$, the following properties hold:

$$
\begin{aligned}
\mathscr{W}_{x} & =\tau_{x} \mathscr{W}_{0}, \\
H_{n}(x) & =\left\langle\mathscr{W}_{0},(t+x)^{n}\right\rangle, \quad n \geq 0,
\end{aligned}
$$

where $\mathscr{W}_{0}$ is symmetric (i.e., $\left.h_{-1}\left(\mathscr{W}_{0}\right)=\mathscr{W}_{0}\right)$, normalized $\left(\right.$ i.e., $\left.\left(\mathscr{W}_{0}\right)_{0}=1\right)$ and satisfying the Pearson equation $\mathscr{W}_{0}^{\prime}-2 t \mathscr{W}_{0}=0$.

Proof. Let $x$ be a fixed real number. We have $\left(\tau_{-x} \mathscr{W}_{x}\right)_{0}=\left(\mathscr{W}_{x}\right)_{0}=H_{0}(x)=1$. If we take (2.4) into account, we can write

$$
\begin{aligned}
\left\langle\left(\tau_{-x} \mathscr{W}_{x}\right)^{\prime}-2 t\left(\tau_{-x} \mathscr{W}_{x}\right), p(t)\right\rangle & =-\left\langle\mathscr{W}_{x}, p^{\prime}(t-x)\right\rangle-2\left\langle\mathscr{W}_{x},(t-x) p(t-x)\right\rangle \\
& =\left\langle\mathscr{W}_{x}^{\prime}, p(t-x)\right\rangle-2\left\langle(t-x) \mathscr{W}_{x}, p(t-x)\right\rangle \\
& =\left\langle\mathscr{W}_{x}^{\prime}-2(t-x) \mathscr{W}_{x}, p(t-x)\right\rangle \\
& =0, \quad p \in \mathbb{P} .
\end{aligned}
$$

So, the normalized linear functional $\tau_{-x} \mathscr{W}_{x}$ satisfies: $\left(\tau_{-x} \mathscr{W}_{x}\right)^{\prime}-2 t\left(\tau_{-x} \mathscr{W}_{x}\right)=0$. The fact that $\mathscr{W}_{0}$ is the unique normalized linear functional satisfying the Pearson equation $\mathscr{W}_{0}^{\prime}-2 t \mathscr{W}_{0}=0$, yields $\tau_{-x} \mathscr{W}_{x}=\mathscr{W}_{0}$ and then $\mathscr{W}_{x}=\tau_{x} \mathscr{W}_{0}$.

Finally, (2.6) follows in a straightforward way from (2.3) and (2.5). 
2.1. An integral representation of $\mathscr{W}_{x}$. At first, we start by giving an integral representation of $\mathscr{W}_{0}$ as follows

$$
\left\langle\mathscr{W}_{0}, p\right\rangle=\int_{-\infty}^{\infty} U(t) p(t) \mathrm{d} t, \quad p \in \mathbb{P}
$$

where we assume that the function $U$ is absolutely continuous on the real line and decaying as fast as its derivative $U^{\prime}$.

By an easy integration by parts, we obtain

$$
\begin{aligned}
0 & =\left\langle\mathscr{W}_{0}^{\prime}-2 t \mathscr{W}_{0}, p\right\rangle=-\left\langle\mathscr{W}_{0}, p^{\prime}(t)+2 t p(t)\right\rangle=-\int_{-\infty}^{\infty} U(t)\left(p^{\prime}(t)+2 t p(t)\right) \mathrm{d} t \\
& =-[U(t) p(t)]_{-\infty}^{\infty}+\int_{-\infty}^{\infty}\left(U^{\prime}(t)-2 t U(t)\right) p(t) \mathrm{d} t, \quad p \in \mathbb{P} .
\end{aligned}
$$

The following condition:

$$
\lim _{t \rightarrow \pm \infty} U(t) p(t)=0, \quad p \in \mathbb{P}
$$

leads to

$$
\int_{-\infty}^{\infty}\left(U^{\prime}(t)-2 t U(t)\right) p(t) \mathrm{d} t=0, \quad p \in \mathbb{P}
$$

This implies

$$
U^{\prime}(t)-2 t U(t)=\lambda f(t),
$$

where $\lambda \neq 0$ is arbitrary and the function $f$ is locally integrable, with rapid decay, and representing the null function, i.e.,

$$
\int_{-\infty}^{\infty} t^{n} f(t) \mathrm{d} t=0, \quad n \geq 0
$$

Conversely, if $U$ is a solution of (2.10) verifying the hypothesis above and the condition:

$$
\int_{-\infty}^{\infty} U(t) \mathrm{d} t \neq 0
$$

then (2.8) and (2.9) are fulfilled and (2.7) defines a linear functional $\mathscr{W}_{0}$, which is a solution of the Pearson equation $\mathscr{W}_{0}^{\prime}-2 t \mathscr{W}_{0}=0$. Putting

$$
f(t)=-\operatorname{sgn}(t) s(|t|), \quad t \in]-\infty,+\infty[,
$$

where $s$ is the Stieltjes function $[10,1,11]$,

$$
s(t)= \begin{cases}0, & t \leq 0 \\ e^{-t^{\frac{1}{4}}} \sin t^{\frac{1}{4}}, & t>0 .\end{cases}
$$


In view of the fact that $\int_{0}^{\infty} t^{n} s(t) \mathrm{d} t=0, n \geq 0$, we get

$$
\begin{aligned}
\int_{-\infty}^{\infty} t^{n} f(t) \mathrm{d} t & =-\int_{-\infty}^{\infty} t^{n} \operatorname{sgn}(t) s(|t|) \mathrm{d} t=\int_{-\infty}^{0} t^{n} s(-t) \mathrm{d} t+\int_{0}^{\infty} t^{n} s(t) \mathrm{d} t \\
& =(-1)^{n} \int_{0}^{\infty} t^{n} s(t) \mathrm{d} t+\int_{0}^{\infty} t^{n} s(t) \mathrm{d} t=\left(1+(-1)^{n}\right) \int_{0}^{\infty} t^{n} s(t) \mathrm{d} t \\
& =0, \quad n \geq 0 .
\end{aligned}
$$

Let $U$ be the function defined on the real line and given by,

$$
\left.U(t)=\lambda e^{t^{2}} \int_{|t|}^{\infty} e^{-y^{2}} s(y) \mathrm{d} y, \quad t \in\right]-\infty,+\infty[.
$$

An easy computation shows that $U^{\prime}(t)=2 t U(t)-\lambda s(t)$ for every $t \geq 0, U^{\prime}(t)=$ $2 t U(t)+\lambda s(-t)$ for every $t<0$.

Equivalently,

$$
\left.U^{\prime}(t)-2 t U(t)=\lambda f(t), \quad t \in\right]-\infty,+\infty[.
$$

For $|t|$ large, we have

$$
|U(t)| \leq|\lambda| e^{t^{2}} \int_{|t|}^{\infty} e^{-y^{2}} e^{-y^{\frac{1}{4}}} \mathrm{~d} y \leq|\lambda| e^{-\frac{1}{2}|t|^{\frac{1}{4}}} e^{t^{2}} \int_{|t|}^{\infty} e^{-y^{2}} \mathrm{~d} y \leq o\left(e^{-\frac{1}{2}|t|^{\frac{1}{4}}}\right), \quad|t| \rightarrow \infty,
$$

by the fact that,

$$
\begin{aligned}
\lim _{|t| \rightarrow \infty} e^{t^{2}} \int_{|t|}^{\infty} e^{-y^{2}} \mathrm{~d} y & =\lim _{x \rightarrow \infty} e^{x^{2}} \int_{x}^{\infty} e^{-y^{2}} \mathrm{~d} y=\lim _{x \rightarrow \infty} \frac{\int_{x}^{\infty} e^{-y^{2}} \mathrm{~d} y}{e^{-x^{2}}}=\lim _{x \rightarrow \infty} \frac{e^{-x^{2}}}{2 x e^{-x^{2}}}=\lim _{x \rightarrow \infty} \frac{1}{2 x} \\
& =0 .
\end{aligned}
$$

Hence, the condition (2.8) holds. Clearly, $\left.U \in L^{1}\right]-\infty,+\infty[$. Condition (2.11) can be written as follows:

$$
\int_{-\infty}^{\infty} U(t) \mathrm{d} t=\lambda S \neq 0
$$

where after reverse the order of integration, we get

$$
\begin{aligned}
S & =2 \int_{0}^{\infty} U(t) \mathrm{d} t=2 \int_{0}^{\infty} e^{t^{2}} \int_{t}^{\infty} e^{-y^{2}} s(y) \mathrm{d} y \mathrm{~d} t \\
& =2 \int_{0}^{\infty} e^{-y^{2}}\left(\int_{0}^{y} e^{t^{2}} \mathrm{~d} t\right) e^{-y^{\frac{1}{4}}} \sin y^{\frac{1}{4}} \mathrm{~d} y,
\end{aligned}
$$

and by making the change of the variable $x=y^{\frac{1}{4}}$, it follows that

$$
S=8 \int_{0}^{\infty} y^{3} e^{-y} F\left(y^{4}\right) \sin y \mathrm{~d} y
$$

where $F(z)=e^{-z^{2}} \int_{0}^{z} e^{t^{2}} \mathrm{~d} t, z \in \mathbb{C}$, is the Dawson function (called also the Dawson integral), [8]. The Dawson function is an entire function for all $z \in \mathbb{C}$ and remains 
bounded for all real number $z$. Recall that the Dawson function satisfies [8]

$$
\begin{aligned}
F(0) & =0, \quad F^{\prime}(z)=-2 z F(z)+1, \quad z \in \mathbb{C}, \\
F(z) & =\sum_{k=0}^{\infty} \frac{(-1)^{k} 2^{k} z^{2 k+1}}{1 \cdot 3 \cdots(2 k+1)}, \quad|z|<\infty \\
F(z) & \simeq \frac{1}{2 z}, \quad|z| \rightarrow \infty \\
F(-z) & =-F(z), \quad z \in \mathbb{C}, \\
0 \leq F(y) & \leq F_{\max }=0,541 \ldots, \quad y \geq 0
\end{aligned}
$$

where $F_{\max }=F\left(x_{\max }\right)$, with $x_{\max }=0,942 \ldots$ Notice that $x_{\max }$ is the only critical point of $F$ on the interval $[0,+\infty$. The following result contains simple but fundamental properties which will be useful in the sequel.

Lemma 2.2. The Dawson function satisfies:

$$
\begin{aligned}
& F(y)<\frac{1}{2 y} \text { if and only if } 0<y<x_{\max }, \\
& F(y)>\frac{1}{2 y} \text { if and only if } y>x_{\max }, \\
& F(y)=\frac{1}{2 y} \text { if and only if } y=x_{\max } .
\end{aligned}
$$

Proof. The proof is an immediate consequence of (2.13) and (2.14).

We can write

$$
S=\int_{0}^{\infty} G(y) \sin y \mathrm{~d} y
$$

where

$$
G(y)=8 y^{3} e^{-y} F\left(y^{4}\right), \quad y \geq 0 .
$$

From (2.16) and (2.14), we obtain

$$
0 \leq G(y) \leq 8 F_{\max } y^{3} e^{-y}, \quad y \geq 0 .
$$

Directly, $G(0)=0$ and $\lim _{y \rightarrow \infty} G(y)=0$, which implies that $G$ has a maximum for $y=\bar{y}>0$, satisfying $G^{\prime}(\bar{y})=0$, i.e.,

$$
F\left(\bar{y}^{4}\right)=\frac{4 \bar{y}^{4}}{8 \bar{y}^{8}+\bar{y}-3} .
$$

Notice that the function $G$ is decreasing on the interval $[\bar{y},+\infty[$.

Lemma 2.3. We have $\bar{y} \leq 3$.

Proof. If we suppose that $\bar{y}>3$, then $F\left(\bar{y}^{4}\right)<\frac{1}{2 \bar{y}^{4}}$. By Lemma 2.2, this yields $\bar{y}^{4}<x_{\max }=0,942 \ldots$, i.e., $\bar{y}<(0,942 \ldots)^{\frac{1}{4}}<3$. This is a contradiction.

Furthermore, the following technical lemma will be needed. 
Lemma 2.4 ([1]). Consider the following integral: $S=\int_{0}^{\infty} G(x) \sin x \mathrm{~d} x$, where the function $G:[0,+\infty[\rightarrow[0,+\infty[$ is continuous on $[0,+\infty[$, decreasing on $[2 \pi,+\infty[$. Suppose that $\int_{0}^{2 \pi} G(y) \sin y \mathrm{~d} y>0$, then $S>0$.

The function $G$ given by (2.16) satisfies the condition of the previous lemma. Indeed, $G$ is a nonnegative function on $[0,+\infty$ [ and decreasing on $[2 \pi,+\infty[$. In order to show that $S$, given by (2.15), is positive, it suffices to prove that $\int_{0}^{2 \pi} G(y) \sin y \mathrm{~d} y>0$. Equivalently,

$$
\int_{0}^{\pi} G(y) \sin y \mathrm{~d} y>-\int_{\pi}^{2 \pi} G(y) \sin y \mathrm{~d} y
$$

In view of Lemma 2.2 , the fact that $G \geq 0, \sin y \geq 0$, for all $y \in[0, \pi], x_{\max }^{\frac{1}{4}}<\frac{\pi}{2}$ and $\sin y \geq \frac{2}{\pi} y$ for all $y \in\left[0, \frac{\pi}{2}\right]$, we obtain

$$
\begin{aligned}
\int_{0}^{\pi} G(y) \sin y \mathrm{~d} y & \geq \int_{x_{\max }^{\frac{1}{4}}}^{\pi} y^{3} e^{-y} \sin y F\left(y^{4}\right) \mathrm{d} y \geq \int_{x_{\max }^{\frac{1}{4}}}^{\pi} \frac{y^{3} e^{-y} \sin y}{2 y^{4}} \mathrm{~d} y \\
& \geq \frac{1}{2} \int_{x_{\max }^{\frac{1}{2}}}^{\frac{\pi}{2}} e^{-y} \frac{\sin y}{y} \mathrm{~d} y \geq \frac{1}{2} \frac{2}{\pi} \int_{x_{\max }^{\frac{1}{4}}}^{\frac{\pi}{2}} e^{-y} \mathrm{~d} y \\
& \geq \frac{1}{\pi}\left(e^{-x_{\max }^{\frac{1}{4}}}-e^{-\frac{\pi}{2}}\right) .
\end{aligned}
$$

Then, we have

$$
\int_{0}^{\pi} G(y) \sin y \mathrm{~d} y \geq \frac{1}{\pi}\left(e^{-x_{\max }^{\frac{1}{4}}}-e^{-\frac{\pi}{2}}\right) \simeq 0,0263 .
$$

On the other hand, we have

$$
-\int_{\pi}^{2 \pi} G(y) \sin y \mathrm{~d} y=-\int_{\pi}^{2 \pi} y^{3} e^{-y} \sin y F\left(y^{4}\right) \mathrm{d} y \leq-F\left(\pi^{4}\right) \int_{\pi}^{2 \pi} y^{3} e^{-y} \sin y \mathrm{~d} y .
$$

By integration by parts and an easy computation we find

$$
-\int_{\pi}^{2 \pi} y^{3} e^{-y} \sin y \mathrm{~d} y=\frac{1}{2} e^{-2 \pi} \pi\left(6+12 \pi+8 \pi^{2}+e^{\pi}\left(3+3 \pi+\pi^{2}\right)\right) \simeq 1,8731
$$

and

$$
F\left(\pi^{4}\right)=e^{-\pi^{8}} \int_{0}^{\pi^{4}} e^{t^{2}} \mathrm{~d} t \leq e^{-\pi^{8}} \int_{0}^{\pi^{4}} e^{\pi^{4} t} \mathrm{~d} t=\frac{1-e^{-\pi^{8}}}{\pi^{4}} \simeq 0,010266
$$

then

$$
-\int_{\pi}^{2 \pi} G(y) \sin y \mathrm{~d} y \leq 1,8731 \cdot 0,010266 \simeq 0,01922 .
$$

From (2.17) and (2.18), we deduce that

$$
\int_{0}^{\pi} G(y) \sin y \mathrm{~d} y>-\int_{\pi}^{2 \pi} G(y) \sin y \mathrm{~d} y .
$$


Proposition 2.1. The normalized Hermite polynomial $H_{n}$ has the following integral representations:

$$
H_{n}(x)=\int_{-\infty}^{\infty} U(t-x) t^{n} \mathrm{~d} t, \quad n \geq 0,|x|<\infty
$$

where

$$
\begin{aligned}
U(t) & =S^{-1} e^{t^{2}} \int_{|t|}^{\infty} e^{-y^{2}} e^{-y^{\frac{1}{4}}} \sin y^{\frac{1}{4}} \mathrm{~d} y, \\
S & =\int_{-\infty}^{\infty} e^{\xi^{2}} \int_{|\xi|}^{\infty} e^{-y^{2}} e^{-y^{\frac{1}{4}}} \sin y^{\frac{1}{4}} \mathrm{~d} y \mathrm{~d} \xi>0 .
\end{aligned}
$$

Proof. It is a straightforward consequence of Lemma 2.1 and 2.4, and (2.12).

2.2. On the zeros of the function $U$. By the change of the variable $y=x^{4}$, the function $U$ given by (2.19), can by written as

$$
U(t)=4 S^{-1} e^{t^{2}} V\left(|t|^{\frac{1}{4}}\right), \quad|t|<\infty,
$$

where

$$
V(t)=\int_{t}^{\infty} x^{3} e^{-x^{8}-x} \sin x \mathrm{~d} x=\int_{0}^{\infty}(x+t)^{3} e^{-(x+t)^{8}-x-t} \sin (x+t) \mathrm{d} x, \quad t \geq 0 .
$$

Clearly, the function $U$ is even and their zeros are exactly those of the function $t \mapsto V\left(|t|^{\frac{1}{4}}\right)$. Observe that we have

$$
V(k \pi)=(-1)^{k} I_{k}, \quad k \geq 0
$$

where

$$
I_{k}=\int_{0}^{\infty} G_{k}(x) \sin x \mathrm{~d} x
$$

and

$$
G_{k}(x)=G_{0}(x+k \pi)=(x+k \pi)^{3} e^{-(x+k \pi)^{8}-(x+k \pi)} .
$$

Lemma 2.5. For every integer $k \geq 0$, we have $I_{k}>0$.

Proof. Let $h(x)=-8 x^{8}-x+3$ for all $x \geq 0$. So, $h^{\prime}(x)=-64 x^{7}-1<0$ for all $x \geq 0$ and $h$ is decreasing on $[0,+\infty[$. The function $h$ is a bijection from $[0,+\infty[$ to $]-\infty, 3]$. Directly, there exists a unique solution $\theta \in[0,+\infty$ [ solution of the equation: $h(x)=0$, where $x \geq 0$. By the intermediate value theorem, we can see that $\frac{1}{2}<\theta<1$, since $h\left(\frac{1}{2}\right)=\frac{9}{2}>0$ and $h(1)=-6<0$. So, $h(x)<0$, for all $\left.x \in\right] \theta,+\infty[$, and $h(x)>0$ for all $x \in\left[0, \theta\left[\right.\right.$. It is clear that $G_{0}^{\prime}(x)=x^{2} e^{-x^{8}-x} h(x)$ for all $x \geq 0$. Thus, $G_{0}$ is decreasing on $[\theta,+\infty[$. The fact that $\theta<1$ allows us to say that:

- the function $G_{0}$ is decreasing on the interval $[\pi,+\infty[$;

- the function $G_{k}$ is decreasing on the interval $[0,+\infty$ [ for every $k \geq 1$.

For every fixed integer $k \geq 1$, we have

$$
I_{k}=\lim _{n \rightarrow \infty} \int_{0}^{2 n \pi} G_{k}(x) \sin x \mathrm{~d} x
$$


Clearly,

$$
\int_{0}^{2 n \pi} G_{k}(x) \sin x \mathrm{~d} x=\sum_{l=0}^{n-1} \int_{0}^{\pi}\left(G_{k}(x+2 l \pi)-G_{k}(x+(2 l+1) \pi)\right) \sin x \mathrm{~d} x,
$$

for every integer $n \geq 1$. Since $\sin x>0$ on $] 0, \pi\left[\right.$, and all the functions $G_{k}, k \geq 1$, are decreasing on $[0,+\infty[$, we have

$$
\int_{0}^{\pi}\left(G_{k}(x+2 l \pi)-G_{k}(x+(2 l+1) \pi)\right) \sin x \mathrm{~d} x>0, \quad l \geq 0 .
$$

Accordingly, it follows that

$$
I_{k} \geq \int_{0}^{\pi}\left(G_{k}(x)-G_{k}(x+\pi)\right) \sin x \mathrm{~d} x>0, \quad k \geq 1 .
$$

For $k=0$, let's note first that $G_{0}$ is nonnegative and continuous on $[0,+\infty[$ and decreasing on $\left[2 \pi,+\infty\left[\right.\right.$. By Lemma 2.4, in order to show that $I_{0}>0$, it suffices to show that $\int_{0}^{2 \pi} G_{0}(x) \sin x \mathrm{~d} x>0$. Equivalently,

$$
\int_{0}^{\pi} G_{0}(x) \sin x \mathrm{~d} x>-\int_{\pi}^{2 \pi} G_{0}(x) \sin x \mathrm{~d} x .
$$

On the one hand, we have

$$
\int_{0}^{\pi} G_{0}(x) \sin x \mathrm{~d} x=\int_{0}^{\theta} G_{0}(x) \sin x \mathrm{~d} x+\int_{\theta}^{\pi} G_{0}(x) \sin x \mathrm{~d} x .
$$

By the fact that $G_{0}(x) \sin x \geq 0$ for every $x \in[0, \pi]$, the function $G_{0}$ is decreasing on the interval $[\theta, \pi]$, we can write

$$
\int_{\theta}^{\pi} G_{0}(x) \sin x \mathrm{~d} x \geq G_{0}(\pi) \int_{\theta}^{\pi} \sin x \mathrm{~d} x=G_{0}(\pi)(1+\cos \theta),
$$

but, $\theta \in] 0, \frac{\pi}{2}[$, then

$$
\int_{\theta}^{\pi} G_{0}(x) \sin x \mathrm{~d} x \geq G_{0}(\pi)=\pi^{3} e^{-\pi^{8}-\pi} .
$$

Since $\theta \in] \frac{\pi}{2}, \pi[\subset] 0, \pi[$, we get

$$
\int_{0}^{\theta} G_{0}(x) \sin x \mathrm{~d} x \geq e^{-\theta^{8}} \int_{0}^{\theta} x^{3} e^{-x} \sin x \mathrm{~d} x \geq e^{-1} \int_{0}^{\frac{\pi}{2}} x^{3} e^{-x} \sin x \mathrm{~d} x,
$$

by an easy computation, we obtain

$$
\int_{0}^{\frac{\pi}{2}} x^{3} e^{-x} \sin x \mathrm{~d} x=\frac{35 \sin \left(\frac{\pi}{2}\right)-19 \cos \left(\frac{\pi}{2}\right)}{16 \sqrt{e}},
$$

and hence,

$$
\int_{0}^{\theta} G_{0}(x) \sin x \mathrm{~d} x \geq \frac{35 \sin \frac{\pi}{2}-19 \cos \frac{\pi}{2}}{16 e \sqrt{e}}=\vartheta,
$$

where $\vartheta \approx 0.0014752$.

From (2.22) and (2.23), we get

$$
\int_{0}^{\pi} G_{0}(x) \sin x \mathrm{~d} x \geq \eta_{1}
$$


where $\eta_{1}=\vartheta+\pi^{3} e^{-\pi^{8}-\pi}$.

On the other hand, since $\sin x \leq 0$, for all $x \in[\pi, 2 \pi]$, we obtain

$$
-\int_{\pi}^{2 \pi} G_{0}(x) \sin x \mathrm{~d} x=-\int_{\pi}^{2 \pi} x^{3} e^{-x^{8}-x} \sin x \mathrm{~d} x \leq-e^{-\pi^{8}} \int_{\pi}^{2 \pi} x^{3} e^{-x} \sin x \mathrm{~d} x,
$$

by an easy computation, we get

$$
-\int_{\pi}^{2 \pi} x^{3} e^{-x} \sin x \mathrm{~d} x=\frac{\pi}{2} e^{-2 \pi}\left(6+12 \pi+8 \pi^{2}+e^{\pi}\left(3+3 \pi+\pi^{2}\right)\right) \approx 1.8731,
$$

and hence,

$$
-\int_{\pi}^{2 \pi} G_{0}(x) \sin x \mathrm{~d} x \leq \eta_{2}
$$

where $\eta_{2}=\beta e^{-\pi^{8}}$ and $\beta \approx 1.8731$.

Since $\eta_{1}>\eta_{2}$, the condition (2.21) holds. Thus, $I_{0}>0$.

Proposition 2.2. The function $U$, given by (2.20), has the following properties.

i) The function $U$ is even and all its zeros are placed symmetrically with respect to the origin.

ii) For every integer $k \geq 0$, $\operatorname{sgn} U\left((k \pi)^{4}\right)=(-1)^{k}$.

iii) For every integer $k \geq 0$, there exists a unique solution $\left.\xi_{k} \in\right](k \pi)^{4},((k+1) \pi)^{4}[$ solution of the equation $U(x)=0$, where $x \in\left[(k \pi)^{4},((k+1) \pi)^{4}\right]$.

Proof. The property given by i) is immediate, by taking (2.20) into account.

By $(2.20), \operatorname{sgn} U(t)=\operatorname{sgn} V\left(t^{\frac{1}{4}}\right)$ for all $t \geq 0$. Since, $V^{\prime}(x)=-t^{3} e^{-t^{8}-t} \sin (t)$ for all $t \geq 0$, then $\operatorname{sgn} V^{\prime}(t)=(-1)^{k+1}$ for all $\left.t \in\right] k \pi,(k+1) \pi[$ and all integer $k \geq 0$. We have already seen that $\operatorname{sgn} V(k \pi)=(-1)^{k}$ for all integer $k \geq 0$. Then, for every integer $k \geq 0$, there exists a unique $\left.\tau_{k} \in\right] k \pi,(k+1) \pi[$ solution to the equation $V(x)=0$, where $x \in[k \pi,(k+1) \pi]$. In view of (2.20), for every integer $k \geq 0$, we infer that $\operatorname{sgn} U\left((k \pi)^{4}\right)=(-1)^{k}$, and there exists a unique $\left.\xi_{k}=\tau_{k}^{4} \in\right](k \pi)^{4},((k+1) \pi)^{4}[$ solution of the equation $U(x)=0$, where $x \in\left[(k \pi)^{4},((k+1) \pi)^{4}\right]$. Thus, ii) and iii) hold.

\section{An Auto-Integral Representation of the Normalized Hermite POLYNOMIALS}

Recall that the Stieltjes integral formula is given by [10]

$$
\int_{0}^{\infty} x^{p-1} e^{-a x} \sin m x \mathrm{~d} x=\frac{\Gamma(p)}{\left(a^{2}+m^{2}\right)^{\frac{p}{2}}} \sin p \theta,
$$

for any positive real numbers $p, q, m$, with $\sin \theta=\frac{m}{r}, \cos \theta=\frac{a}{r}, 0<\theta<\frac{\pi}{2}$ and $r=\sqrt{a^{2}+m^{2}}$. 
From (3.1) taking with $\theta=\frac{\pi}{4}$, it comes that $a=m=1$, and

$$
\int_{0}^{\infty} x^{p-1} e^{-x} \sin x \mathrm{~d} x=\frac{\Gamma(p)}{2^{\frac{p}{2}}} \sin \frac{p \pi}{4}, \quad p>0 .
$$

In particular, for $p=4(n+1)$, we get

$$
\int_{0}^{\infty} x^{4 n+3} e^{-x} \sin x \mathrm{~d} x=0, \quad n \geq 0
$$

and the transformation $x=t^{\frac{1}{4}}$, yields,

$$
\int_{0}^{\infty} t^{n} e^{-t^{\frac{1}{4}}} \sin t^{\frac{1}{4}} \mathrm{~d} t=0, \quad n \geq 0 .
$$

On the other hand, by (3.1) and the recursion property of the Gamma function, to known $\Gamma(z+1)=z \Gamma(z)$, for all $z \in \mathbb{C}$ such that $z \neq-n$ for every integer $n \geq 0$, and $\Gamma(1)=1$, we can write

$$
\int_{0}^{\infty} x^{p-1} e^{-a x} \sin m x \mathrm{~d} x=\frac{\Gamma(p+1)}{\left(a^{2}+m^{2}\right)^{\frac{p}{2}}} \frac{\sin p \theta}{p}, \quad p>0,
$$

and by letting $p \rightarrow 0^{+}$, we get

$$
\int_{0}^{\infty} \frac{e^{-a x} \sin m x}{x} \mathrm{~d} x=\theta
$$

For $\theta=\frac{\pi}{4}, m=a=1$, the transformation $x=t^{\frac{1}{4}}$, gives us

$$
\int_{0}^{\infty} \frac{e^{-t^{\frac{1}{4}}} \sin t^{\frac{1}{4}}}{\pi t} \mathrm{~d} t=1
$$

and by taking (3.2) into account, we obtain

$$
\int_{-\infty}^{\infty} t^{n} W(t) \mathrm{d} t=\delta_{n, 0}, \quad n \geq 0
$$

where

$$
W(t)= \begin{cases}\frac{e^{-t^{\frac{1}{4}}} \sin t^{\frac{1}{4}}}{\pi t}, & \text { if } t>0 \\ 0, & \text { if } t \leq 0 .\end{cases}
$$

This leads to the following integral representation of the Dirac mass $\delta_{0}$,

$$
\left\langle\delta_{0}, p\right\rangle=\int_{-\infty}^{\infty} W(t) p(t) \mathrm{d} t=p(0), \quad p \in \mathbb{P}
$$

and more general to an integral representation of the Dirac mass $\delta_{x}$, for every real number $x$,

$$
\left\langle\delta_{x}, p\right\rangle=\int_{-\infty}^{\infty} W(t-x) p(t) \mathrm{d} t=p(x), \quad p \in \mathbb{P} .
$$

Consequently, the following auto-integral representation of the normalized $\mathcal{H}$ ermite polynomial $H_{n}$ holds

$$
H_{n}(x)=\int_{-\infty}^{\infty} W(t-x) H_{n}(t) \mathrm{d} t, \quad n \geq 0 .
$$




\section{REFERENCES}

[1] K. Ali Khelil, R. Sfaxi and A. Boukhemis, Integral representation of the generalized Bessel linear functional, Bull. Math. Anal. Appl. 9(3) (2017), 1-15.

[2] N. I. Akhiezer, The Classical Moment Problem, Oliver and Boyd, Edinburgh, London, 1965.

[3] T. S. Chihara, An Introduction to Orthogonal Polynomials, Gordon and Breach, New York, 1978.

[4] A. Ghressi and L. Kheriji, Some new results about a symmetric D-semiclassical linear form of class one, Taiwanese J. Math. 11(2) (2007), 371-382.

[5] M. E. H. Ismail and D. Stanton, Classical orthogonal polynomials as moments, Canad. J. Math. 49 (1997), 520-542.

[6] M. E. H. Ismail and D. Stanton, More orthogonal polynomials as moments, in: Mathematical Essays in Honor of Gian-Carlo Rota, Cambridge, MA, 1996, Birkhäuser Boston, Boston, MA, 1998, 377-396.

[7] M. E. H. Ismail and D. Stanton, q-Integral and moment representations for q-orthogonal polynomials, Canad. J. Math. 45 (2002), 709-735.

[8] N. N. Lebedev, Special Functions and their Applications, Translated from the Russian by Richard A. Silverman, Englewood Cliffs, New Jork, 1965.

[9] P. Maroni, Une théorie algébrique des polynômes orthogonaux, Applications aux polynômes orthogonaux semi-classiques, IMACS: International Association for Mathematics and Computers in Simulation 9 (1991), 95-130.

[10] P. Maroni, Fonctions eulériennes, polynémes orthogonaux classiques, Techniques de l'Ingénieur 154 (1994), 1-30.

[11] P. Maroni, An integral representation for the Bessel form, J. Comput. Appl. Math. 157 (1995), 251-260.

[12] M. Rahman and A. Verma, A q-integral representation for the Rogers q-ultraspherical polynomials and some applications, Constr. Approx. 2 (1986), 1-10.

[13] J. Shohat and J. D. Tamarkin, The Problem of Moments, American Mathematical Society, Providence, 1950.

[14] B. Simon, The classical moment as a selfadjoint finite difference operator, Adv. Math. 137 (1998), 82-203.

[15] G. Szegö, Orthogonal Polynomials, Fourth Edition, American Mathematical Society, Providence, 1975.

${ }^{1}$ Department of Mathematics, UNIVERSITY OF BADJI MOKHTAR, Annaba, Algeria

Email address: aboukhemis@yahoo.com

Email address: kalikhelil@gmail.com

${ }^{2}$ Coll. Edu. of Girls, Scientific Sections, UNIVERSITY OF HAFR-AL-BATIN,

Hafr-Al-Batin, Saudi Arabia

Email address: ridhasfaxi@gmail.com 